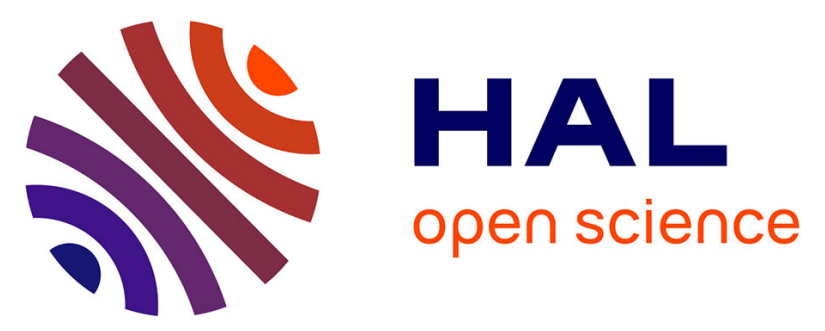

\title{
Temperature dependence of the zero-phonon linewidth in quantum dots: An effect of the fluctuating environment
}

Ivan Favero, Alice Berthelot, Guillaume Cassabois, Christophe Voisin, Claude

Delalande, Philippe Roussignol, Robson Ferreira, Jean-Michel Gérard

\section{To cite this version:}

Ivan Favero, Alice Berthelot, Guillaume Cassabois, Christophe Voisin, Claude Delalande, et al.. Temperature dependence of the zero-phonon linewidth in quantum dots: An effect of the fluctuating environment. Physical Review B: Condensed Matter and Materials Physics (1998-2015), 2007, 75, pp.073308. hal-00284909

\section{HAL Id: hal-00284909 \\ https://hal.science/hal-00284909}

Submitted on 4 Jun 2008

HAL is a multi-disciplinary open access archive for the deposit and dissemination of scientific research documents, whether they are published or not. The documents may come from teaching and research institutions in France or abroad, or from public or private research centers.
L'archive ouverte pluridisciplinaire HAL, est destinée au dépôt et à la diffusion de documents scientifiques de niveau recherche, publiés ou non, émanant des établissements d'enseignement et de recherche français ou étrangers, des laboratoires publics ou privés. 


\title{
Temperature-dependence of the zero-phonon line-width in quantum dots: an effect of the fluctuating environment
}

\author{
I. Favero ${ }^{1}$, A. Berthelot ${ }^{1}$, G. Cassabois ${ }^{1, *}$, C. Voisin ${ }^{1}$, C. Delalande ${ }^{1}$, Ph. Roussignol $^{1}$, R. Ferreira ${ }^{1}$ and J. M. Gérard $^{2}$ \\ ${ }^{1}$ Laboratoire Pierre Aigrain, Ecole Normale Supérieure, 24 rue Lhomond 75231 Paris Cedex 5, France \\ ${ }^{2}$ CEA-CNRS-UJF "Nanophysics and Semiconductors" Laboratory, \\ CEA/DRFMC/SP2M, 17 rue des Martyrs 38054 Grenoble Cedex 9, France
}

(Dated: December 19, 2006)

\begin{abstract}
We report systematic measurements on the broadening of the emission spectrum of single quantum dots as a function of temperature and incident power. Spectral diffusion effects in the motional narrowing regime provide a quantitative interpretation of our experimental results. We show that, at low incident power, the thermal activation of spectral diffusion results in a Lorentzian zero-phonon line with a width that increases linearly with temperature. Our study provides an unified interpretation to the widely debated issue of the dispersion of the data on the temperature dependence of this zero-phonon line-width.
\end{abstract}

PACS numbers: 78.67.Hc, 78.55.Cr

The optical spectrum of semiconductor quantum dots (QDs) has recently attracted considerable interest because of its importance for fundamental physics and applications in quantum information processing. Two spectroscopic features have been particularly addressed, namely the optical lineshape in the zero-temperature limit and the temperature-induced broadening of the QD spectrum. In the former case, the experimental results show marked differences depending on the spectroscopic techniques. In photoluminescence (PL) measurements, the experimental evidence of light-induced spectral diffusion in single QDs has revealed the existence of randomly fluctuating electric fields due to the presence of localized charges in the vicinity of the QD [1-4]. This phenomenon with spectral jitters ranging from few tens of $\mu \mathrm{eV}$ to several meV breaks down the simple picture of a two-level system where the linewidth is given by the radiative limit in the $\mu \mathrm{eV}$ range. However, spectral diffusion effects appear to be considerably reduced under resonant excitation of the fundamental interband transition and the limit of radiatively-limited optical spectra is nearly reached either in non-linear four-wave mixing experiments [5] or in linear absorption measurements [6].

The temperature-induced broadening of the optical spectrum is an other major issue of the QD optical properties. Temperature-dependent measurements have shown that the line-shape can be described by the superposition of the so-called zero-phonon line (ZPL) and broad side-bands extending on several meV [7-9]. These side-bands arise from the radiative recombination of electron-hole pairs assisted by the emission and the absorption of acoustic phonons. This pure dephasing phenomenon is interpreted in the framework of the HuangRhys theory by an independent boson model with a linear coupling to acoustic phonons. However, within this theoretical treatment, the ZPL does not show any broadening with temperature in strong contrast with published data [10-13]. In a more sophisticated model including a quadratic coupling to acoustic phonons via phononassisted transitions to higher QD levels [14], it has been shown that the ZPL width increases linearly with temperature [15]. Nevertheless, the magnitude of this contribution can not quantitatively explain the experimental results [10-13], and in particular the confinement energy dependence found in Ref. [13]. Phonon damping by scattering at surfaces also results in a linear increase with temperature of the ZPL width and this process was proposed in Ref. [12] to explain the measurements carried out on different mesa sizes. However, again, the magnitude of this contribution is too small to account for the experimental results in Ref. [11] where single QD spectroscopy is performed without mesas. The dispersion of the data on the temperature dependence of the ZPL width suggests the existence of an additional contribution. Such a process is missing in the different models developed in Ref. $[12,15]$ which both rely on an interaction between acoustic phonons and carriers confined inside the QDs.

The recent experimental evidence of a motional narrowing regime for the spectral diffusion phenomenon in QDs [16] shines a new light on the two former issues that are separately debated in the literature. In Ref. [16] two regimes of fluctuation are considered for the electrostatic environment, namely the slow modulation limit where the optical line-shape reflects directly the statistical distribution of the fluctuating system energy, and the fast modulation one (or motional narrowing) where the fluctuation is smoothed out and the line-shape is narrowed into a Lorentzian profile. The motional narrowing regime was shown to take place surprisingly at low temperature thus explaining why the ZPL may have a Lorentzian profile with a width which is not given by the intrinsic radiative limit, but by the extrinsic reservoir fluctuation dynamics.

In this paper, we show that the thermal activation of the processes leading to spectral diffusion in the motional narrowing regime results in a Lorentzian ZPL with a width that increases linearly with temperature. This contribution to the temperature dependence of the ZPL width relies on an interaction between acoustic phonons and carriers outside the QDs. This extrinsic dephas- 
ing process is studied by systematic measurements of the linewidth for single QDs in the motional narrowing regime, i.e. with a ZPL that keeps a Lorentzian profile in the investigated range of experimental parameters. The importance of this extrinsic contribution to the dephasing processes at low temperature is further illustrated by the example of a peculiar QD where the efficiency of the temperature-induced broadening can abruptly change from one day to the other.

We study self-assembled InAs/GaAs QDs grown by molecular beam epitaxy in the Stranski-Krastanow mode. Sub- $\mu \mathrm{m}$ mesa patterns are processed on the sample surface in order to isolate single QDs [17]. The QDs studied in this paper come from the same wafer as in Ref. [16] and they are located in $0.5 \mu \mathrm{m}$ mesas. SingleQD spectroscopy at low temperature is carried out by standard micro-PL measurements in the far field using a microscope objective (numerical aperture 0.5) in a confocal geometry. The excitation beam is provided by a He:Ne laser and we perform PL measurements under nonresonant excitation at $1.96 \mathrm{eV}$. Both shape and width of the emission line of single QDs are analyzed by highresolution Fourier-transform spectroscopy [18].

In Fig. 1(b) we display the full width at half maximum (FWHM) of the PL spectrum of a single QD, as a function of incident power for three temperatures $(20$, 30 and $40 \mathrm{~K})$. The emission line is centered at $1.375 \mathrm{eV}$ at $10 \mathrm{~K}$, and its profile remains Lorentzian in the investigated range of incident power and temperature, as can be seen in Fig. 1(a) from the exponential decay of the interferogram contrasts $C(\tau)$ recorded by Fourier-transform spectroscopy [18]. This behavior contrasts with the one reported in Ref. [16] where a smooth transition between Lorentzian and Gaussian profiles was observed on increasing incident power or temperature. This means that the spectral diffusion phenomenon is always in the motional narrowing regime in the present study, because of a smaller modulation amplitude $\Sigma_{s}$ as detailed below. In Fig. 1(b), at 20K, the ZPL width increases by a factor of 4 over the two decades of incident power, with a pronounced rise around $0.6 \mathrm{~kW} . \mathrm{cm}^{-2}$. At higher temperatures, the variations are qualitatively the same but the ZPL broadening with incident power is reduced when the temperature increases. The ZPL width increases only by a factor of 2.5 at $30 \mathrm{~K}$, and 1.7 at $40 \mathrm{~K}$. The systematic measurements displayed in Fig. 1(b) indicate in fact the existence of two regimes. In the first one, at low incident power $\left(\mathrm{P}<0.2 \mathrm{~kW} . \mathrm{cm}^{-2}\right)$, the temperature dependence of the ZPL consists of a linear increase with temperature (Fig. 1(b), inset), that can be described by the simple expression:

$$
\Gamma=\Gamma_{0}+\alpha_{0} T
$$

where the slope $\alpha_{0}$ characterizes the efficiency of the temperature-induced broadening and hardly depends on the excitation density. For the QD discussed in Fig. 1 we find for $\alpha_{0}$ a value of $1.55 \pm 0.15 \mu \mathrm{eV} . \mathrm{K}^{-1}$ (Fig. 1(b), inset), i.e. a standard figure compared to published data, where $\alpha_{0}$ ranges between 0.05 and $4 \mu \mathrm{eV} . \mathrm{K}^{-1}$ [10-13]. In the second regime, at high incident power $\left(\mathrm{P}>0.2 \mathrm{~kW} . \mathrm{cm}^{-2}\right)$, the efficiency of the temperatureinduced broadening does depend on the incident power $\left(\Gamma=\Gamma_{0}(P)+\alpha(P) T\right)$, and the higher the excitation density the smaller the ZPL broadening with temperature $\left(\alpha(P) \leqslant \alpha_{0}\right)$. For $\mathrm{P} \gtrsim 2 \mathrm{~kW} \cdot \mathrm{cm}^{-2}$ the variations lie within our experimental uncertainty. We obtain an upper-limit of $0.25 \mu \mathrm{eV} . \mathrm{K}^{-1}$ for the $\alpha(P)$ coefficient at high incident power.

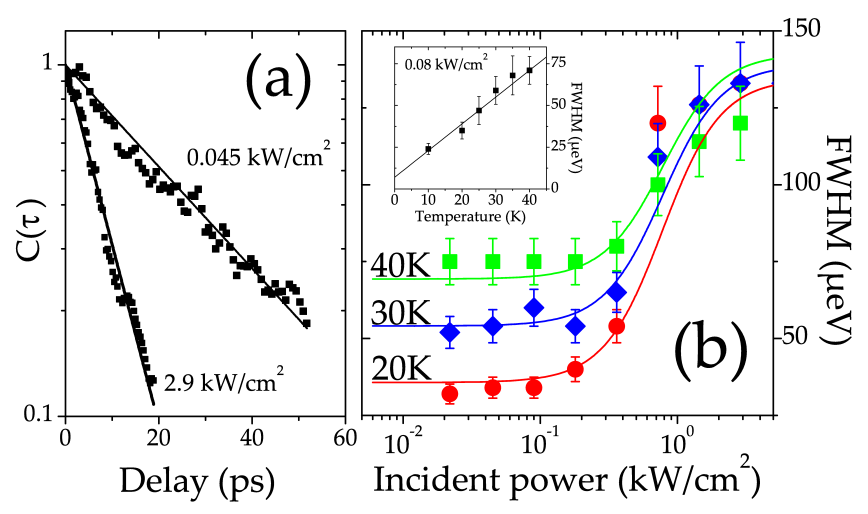

FIG. 1: (a) Fourier-transform spectroscopy on the PL signal of a single QD at $20 \mathrm{~K}$, for two incident powers. Exponential fits (solid lines) with a time constant $T_{2} \sim 30$ ps at 0.045 $\mathrm{kW} . \mathrm{cm}^{-2}$, and $8.5 \mathrm{ps}$ at $2.9 \mathrm{~kW} \cdot \mathrm{cm}^{-2}$. (b) Full width at half maximum (FWHM) of the zero-phonon line, as a function of incident power for three temperatures: 20K (red circles), 30K (blue diamonds) and 40K (green squares). The solid lines (20K in red, $30 \mathrm{~K}$ in blue, $40 \mathrm{~K}$ in green) are calculated within a model taking into account the carrier dynamics in the environment (Eq. (2)\&(3)). Inset: Temperature dependence of the FWHM of the zero-phonon line for an incident power of $0.08 \mathrm{~kW} . \mathrm{cm}^{-2}$. The solid line is a linear regression of the data with a slope $\alpha_{0} \sim 1.55 \mu \mathrm{eV} . \mathrm{K}^{-1}$.

From the analysis of the data of Fig. 1, we conclude that the ZPL broadening can not be characterized by two uncorrelated, additive contributions $\Delta \Gamma(T)$ and $\Delta \Gamma(P)$ that would depend only on temperature and incident power, respectively. We provide now a quantitative interpretation of these experimental results in the framework of the motional narrowing phenomenon for the spectral diffusion effect [16].

The optical spectrum of semiconductor QDs is highly sensitive to the presence of fluctuating electric fields. Impurities, defects or localized charges in the vicinity of a QD induce micro-electric fields that shift the QD emission line through the quantum confined Stark effect [1-4]. The fluctuations of the QD environment thus randomize the emission energy over a spectral range $\Sigma$ on a characteristic correlation time $\tau_{c}$. In Ref. [16], a crossover from Lorentzian to Gaussian profiles of the ZPL was observed on increasing either the incident power or the temperature. This effect indicated a smooth transition between 
the two regimes of fluctuation, namely the fast modulation one (or motional narrowing) where the ZPL profile was Lorentzian with $\Sigma \tau_{c}$ decreasing down to $0.4 \hbar$, and the slow modulation one where the ZPL profile was Gaussian with $\Sigma \tau_{c}$ increasing up to $1.35 \hbar$. In the present study, we are always in the motional narrowing regime, and the FWHM of the Lorentzian ZPL is given by $2 \Sigma^{2} \tau_{c} / \hbar$.

The fluctuating environment can be described in a simple model where $N$ uncorrelated traps individually induce a Stark shift $\Delta$ of the QD emission line. The carrier dynamics in the traps is characterized by a capture time $\tau_{\downarrow}$ and an escape time $\tau_{\uparrow}$. The correlation time $\tau_{c}$ and the spectral modulation amplitude $\Sigma$ are then given by the expressions $1 / \tau_{c}=1 / \tau_{\uparrow}+1 / \tau_{\downarrow}$ and $\Sigma=2 \Sigma_{s} /\left(\sqrt{\tau_{\uparrow} / \tau_{\downarrow}}+\sqrt{\tau_{\downarrow} / \tau_{\uparrow}}\right)$, respectively, where the maximum value $\Sigma_{s}$ of the modulation amplitude is reached for $\tau_{\downarrow} \sim \tau_{\uparrow}$ and is equal to $\sqrt{N} \Delta / 2$ [16].

We now compare the predictions of this model with our systematic measurements of the ZPL width as a function of temperature and incident power. We plot in solid lines in Fig. 1(b) the theoretical values of the FWHM of the ZPL that are calculated by assuming that:

$$
\begin{aligned}
\frac{1}{\tau_{\downarrow}} & =\frac{1}{\tau_{1}}\left(1+n_{1}(T)\right)+\frac{1}{\tau_{2}}\left(1+n_{2}(T)\right) \\
\frac{1}{\tau_{\uparrow}} & =\frac{1}{\tau_{1}} n_{1}(T)+\frac{1}{\tau_{2}} n_{2}(T)+\frac{1}{\tau_{3}} f(P)
\end{aligned}
$$

The existence of phonon-assisted mechanisms is taken into account by the first two terms on the right handside of Eq. (2)\&(3). The capture (escape) is thermallyactivated due to the emission (absorption) of an acoustic phonon of mean energy $E_{1}$ or an optical phonon of energy $E_{2}$. The capture and escape efficiencies are proportional to $\left(1+n_{i}(t)\right)$ and $n_{i}(t)$ respectively, where $n_{i}(t)$ is a BoseEinstein occupation factor given by $1 /\left(\exp \left(E_{i} / k_{B} T\right)-1\right)$. In Eq. (3) the additional term accounts for the contribution of Auger processes to carrier escape. $f(P)$ has the general form $P^{\beta} /\left(P^{\beta}+P_{0}^{\beta}\right)$, where $\beta$ indicates the power law exponent for the dependence of the Auger processes on incident power, and $P_{0}$ is the excitation density where saturation of the Auger processes appears [19].

In Fig. 1(b), we observe a fair agreement between the data and the calculations for the set of parameters $\Sigma_{s} \sim 150 \mu \mathrm{eV}, \tau_{1} \sim 200 \mathrm{ps}, \tau_{2} \sim 5 \mathrm{ps}, \tau_{3} \sim 35 \mathrm{ps}, E_{1} \sim 1 \mathrm{meV}$, $E_{2} \sim 30 \mathrm{meV}, \beta=2$, and $P_{0} \sim 0.96 \mathrm{~kW} . \mathrm{cm}^{-2}$. Note that the inclusion of the same Auger term $f(P) / \tau_{3}$ for the capture mechanism in Eq. (2), although physically sound, hardly modifies our theoretical calculations and almost identical fits are obtained by increasing $\Sigma_{s}$ by $10 \%$. As far as the determination of the time constants $\tau_{1}, \tau_{2}$ and $\tau_{3}$ is concerned, the theoretical calculations displayed in Fig. 1(b) depend only on the ratio $\tau_{1} / \tau_{2}$ and $\tau_{3} / \tau_{2}$. An estimation of the absolute value of $\tau_{2}$ would be possible if a crossover from Lorentzian to Gaussian lineshapes occurred. Since $\tau_{2}$ is the smallest time constant, the correlation time $\tau_{c}$ is given by $\tau_{2}$ in a first approximation, and its value could be estimated from the FWHM of the ZPL at the Lorentzian-Gaussian crossover where the product of the modulation amplitude $\Sigma$ and correlation time $\tau_{c}$ is close to $\hbar[16]$. In the present study where the ZPL keeps a Lorentzian profile, we are always in the motional narrowing regime and the condition $\Sigma \tau_{c}<\hbar$ must be fulfilled in our calculations. This inequality is verified if $\tau_{2}$ is smaller than the upper bound of $5 \mathrm{ps}$, and in Fig. 1(b), $\Sigma \tau_{c} \leqslant 0.7 \hbar$ which ensures that the ZPL is Lorentzian.

If we now examine the relative importance of the microscopic processes involved in the carrier dynamics in the QD environment, we observe that the capture process is dominated by the emission of optical phonon, and the escape one is activated either by acoustic phonon absorption on increasing the temperature, or by Auger processes on raising the excitation density. This hierarchy between the different mechanisms is qualitatively the same as the one deduced in Ref. [16] where it was pointed out that the capture process is indeed much more efficient than the escape one. However, quantitatively, we notice that in the present case the asymmetry between the capture and escape processes is less pronounced than in Ref. [16]. In the latter study, the ratio between the capture and escape times $\tau_{\downarrow} / \tau_{\uparrow}$ was always smaller than $10^{-2}$ whereas, here, it increases up to 0.2 . This difference is in fact at the origin of the dependence of the $\alpha$ coefficient on incident power $\left(\Gamma=\Gamma_{0}(P)+\alpha(P) T\right)$. In the limit where $\tau_{\downarrow} / \tau_{\uparrow} \ll 1$, the modulation amplitude $\Sigma$ increases linearly with $\sqrt{\tau_{\downarrow} / \tau_{\uparrow}}$ and $\tau_{c} \sim \tau_{\downarrow}$, so that the FWHM of the ZPL is given by:

$$
\Gamma \sim 8\left(\frac{\Sigma_{s} \tau_{\downarrow}}{\hbar}\right)^{2} \frac{\hbar}{\tau_{\uparrow}}
$$

where the contributions of the phonon-assisted and Auger processes become separable according to Eq. (3). This situation corresponds to the regime of low excitation density in Fig. $1\left(\mathrm{P}<0.2 \mathrm{~kW} . \mathrm{cm}^{-2}\right)$, and from Eq. (4), we easily derive the expression of the $\alpha_{0}$ coefficient:

$$
\alpha_{0}=\frac{8 \Sigma_{s}^{2} \tau_{\downarrow}^{2} k_{B}}{\hbar \tau_{1} E_{1}}
$$

where $k_{B}$ is the Boltzmann constant. Since the capture process is dominated by optical phonon emission $\left(\tau_{\downarrow} \sim \tau_{2}\right)$, a numerical evaluation of Eq. (5) with $\tau_{\downarrow} \sim 5$ ps gives a $\alpha_{0}$ coefficient of $1.47 \mu \mathrm{eV} . \mathrm{K}^{-1}$, that is consistent with our experimental data (Fig. 1(b), inset).

On the other hand, when $\tau_{\downarrow} / \tau_{\uparrow} \lesssim 1$, the modulation amplitude $\Sigma$ deviates from its asymptotic behavior $2 \Sigma_{s} \sqrt{\tau_{\downarrow} / \tau_{\uparrow}}$ and the linear approximation of Eq. (4) is no longer valid. As a matter of fact, when $\tau_{\downarrow} / \tau_{\uparrow}$ increases, $\partial \Sigma / \partial\left(\tau_{\downarrow} / \tau_{\uparrow}\right)$ decreases and tends to zero for $\tau_{\downarrow} / \tau_{\uparrow} \sim 1$, where the modulation amplitude $\Sigma$ reaches its saturation value $\Sigma_{s}$. In other words, the temperature-induced broadening becomes less and less efficient when increasing the incident power, which explains the dependence on power of the $\alpha$ coefficient and its decrease as a function of excitation density (Fig. 1). 
From the expression of $\alpha_{0}$ in Eq. (5), we thus conclude that the carrier dynamics in the QD environment provides a significant contribution to the temperature-induced dephasing in the low temperature limit $(\mathrm{T}<50 \mathrm{~K})$. In contrast to the model proposed in Ref. $[12,15]$, the temperature-dependence of the ZPL width comes from the interaction of acoustic phonons with carriers outside the QD. This purely extrinsic contribution suggests the picture of the decoherence dynamics in QDs as a probe of the carrier dynamics in the QD environment and provides a simple interpretation to the wide dispersion of the published experimental data on the ZPL broadening [10-13]. We have even observed that $\alpha_{0}$ varies from QD to QD all located in the same mesa, thus indicating that the scale of the fluctuating environment is smaller than the mesa size $(0.5 \mu \mathrm{m}$ here $)$.

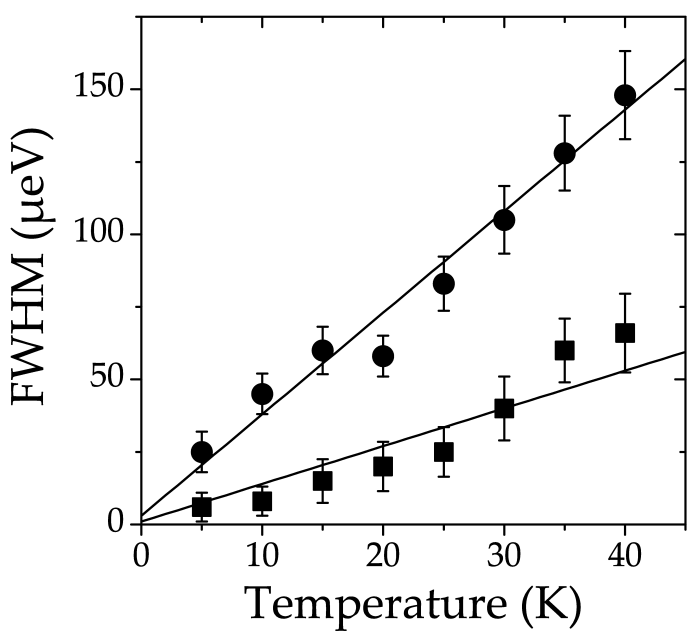

FIG. 2: Temperature dependence of the full width at half maximum (FWHM) of the zero-phonon line of a single QD (different from the one in Fig. 1), for two different days.

We further illustrate the extrinsic origin of the temper- ature dependence of the ZPL broadening by the striking example of another QD where the $\alpha_{0}$ coefficient shows abrupt changes from one day to another. In Fig. 2 we display the measurements of the ZPL width of this QD, at low incident power, for two different days of acquisition. By two different days, we mean that the sample has been heated up to room temperature and cooled down to $10 \mathrm{~K}$ at least one time between the two runs. The fits displayed in solid lines are performed according to Eq. (1) and we find $\Gamma_{0}=1 \mu \mathrm{eV}, \alpha_{0}=1.3 \mu \mathrm{eV} . \mathrm{K}^{-1}$ and $\Gamma_{0}=3 \mu \mathrm{eV}, \alpha_{0}=3.5 \mu \mathrm{eV} . \mathrm{K}^{-1}$ for the two sets of data. We stress that we have only observed these two values for the $\alpha_{0}$ coefficient, and that these measurements have been obtained several times, at low incident power, either under non-resonant excitation at $1.96 \mathrm{eV}$ or under quasi-resonant excitation of the wetting-layer continuum at $1.43 \mathrm{eV}$. Since the PL experiments have been carefully carried out in the low-excitation limit, we can rule out any incident power effect to explain the variations of $\alpha_{0}$. We suggest the existence of two sub-classes of traps of amounts $N_{1}$ and $N_{2}$ where one type of traps is completely inactive depending on the cooling cycle. This effect would result in day-by-day fluctuations of the saturation value $\Sigma_{s}$ which modifies the efficiency of the temperature-induced broadening (Eq. (5)).

In conclusion, we report systematic measurements of the ZPL width of single QDs as a function of temperature and incident power. We interpret our experimental results by spectral diffusion effects in the motional narrowing regime. Our study shows that the carrier dynamics in the QD environment provides a significant contribution to the temperature-induced dephasing in the low temperature limit, and provides an unified interpretation to the widely debated issue of the dispersion of the data on the linear temperature dependence of the ZPL width.

*e-mail: Guillaume.Cassabois@lpa.ens.fr

LPA-ENS is "unité mixte (UMR 8551) de l'ENS, du CNRS, des Universités Paris 6 et 7". This work is financially supported by the region Ile de France through the project SESAME E-1751.
[1] S. A. Empedocles, D. J. Norris, and M. G. Bawendi, Phys. Rev. Lett. 77, 3873 (1996).

[2] H. D. Robinson and B. B. Goldberg, Phys. Rev. B 61, R5086 (2000).

[3] V. Türck, S. Rodt, O. Stier, R. Heitz, R. Engelhardt, U. W. Pohl, D. Bimberg, and R. Steingrüber, Phys. Rev. B 61, 9944 (2000).

[4] L. Besombes, K. Kheng, L. Marsal, and H. Mariette, Phys. Rev. B 65, 121314 (2002).

[5] W. Langbein, P. Borri, U. Woggon, V. Stavarache, D. Reuter, and A. D. Wieck, Phys. Rev. B 70, 033301 (2004).

[6] A. Högele, S. Seidl, M. Kroner, K. Karrai, R. J. Warburton, B. D. Gerardot, and P. M. Petroff, Phys. Rev. Lett.
93, $217401(2004)$

[7] L. Besombes, K. Kheng, L. Marsal, and H. Mariette, Phys. Rev. B 63, 155307 (2001)

[8] P. Borri, W. Langbein, S. Schneider, U. Woggon, R. L. Sellin, D. Ouyang, and D. Bimberg, Phys. Rev. Lett. 87, 157401 (2001).

[9] I. Favero, G. Cassabois, R. Ferreira, D. Darson, C. Voisin, J. Tignon, C. Delalande, G. Bastard, Ph. Roussignol, and J. M. Gérard, Phys. Rev. B 68, 233301 (2003).

[10] C. Kammerer, C. Voisin, G. Cassabois, C. Delalande, Ph. Roussignol, F. Klopf, J. P. Reithmaier, A. Forchel, and J. M. Gérard, Phys. Rev. B 66, 041306 (2002).

[11] B. Urbaszek, E. J. McGhee, M. Krüger, R. J. Warburton, K. Karrai, T. Amand, B. D. Gerardot, P. M. Petroff, and 
J. M. Garcia, Phys. Rev. B 69, 035304 (2004).

[12] G. Ortner, D. R. Yakovlev, M. Bayer, S. Rudin, T. L. Reinecke, S. Fafard, Z. Wasilewski, and A. Forchel, Phys. Rev. B 70, 201301 (2004).

[13] P. Borri, W. Langbein, U. Woggon, V. Stavarache, D. Reuter, and A. D. Wieck, Phys. Rev. B 71, 115328 (2005).

[14] T. Takagahara, Phys. Rev. B 60, 2638 (1999).

[15] E. A. Muljarov and R. Zimmermann, Phys. Rev. Lett. 93, 237401 (2004)

[16] A. Berthelot, I. Favero, G. Cassabois, C. Voisin, C. De- lalande, Ph. Roussignol, R. Ferreira, and J. M. Gérard, Nature Phys. 2, 759 (2006).

[17] J. Y. Marzin, J. M. Gérard, A. Izraël, D. Barrier, and G. Bastard, Phys. Rev. Lett. 73, 716 (1994).

[18] C. Kammerer, G. Cassabois, C. Voisin, M. Perrin, C. Delalande, Ph. Roussignol, and J. M. Gérard, Appl. Phys. Lett. 81, 2737 (2002).

[19] U. Bockelmann and T. Egeler, Phys. Rev. B 46, 15574 (1992). 